M2

\title{
GENETICS OF GILLES DE LA TOURETTE SYNDROME
}

\author{
K.K. Kidd', A.J. Pakstis', P. Heutink4, D.L. Pauls ${ }^{2,1}$, R. Kurlan ${ }^{3}$, J. Weber, P. Wilkie, B.J.M. van de Wetering', and B.A. Oostra \\ Dept. of Genetics ${ }^{1}$ and Child Study Center ${ }^{2}$, Yale University School of Medicine, New Haven, CT, USA; Dept. of Neurology ${ }^{3}$, University of Rochester \\ Medical Center, Rochester, NY, USA; Depts. of Clinical Genetics and Psychiatry', Erasmus University and University Hospital, Rotterdam-Dijkzigt, \\ The Netherlands; Marshfield Clinic ${ }^{6}$, Marshfield Medical Research Foundation, Marshfield, WI, USA
}

Gilles de la Tourette Syndrome (TS) is a familial, neuropsychiatric disorder characterized by chronic, intermittent motor and vocal tics that typically appear in childhood although the clinical expression varies. Affected individuals may display additional symptoms, but the diagnosis is based on the presence of both motor and vocal tics. Evidence suggests that obsessive compulsive disorder (OCD) and chronic multiple tics (CT) alone (motor not vocal) can sometimes be alternate expressions of the same underlying disorder as TS. Attention deficit hyperactivity disorder (ADHD) has been thought by some researchers to be related to TS but this has not been clearly established.

Research on twins and adoption studies support a genetic basis for the disorder. Segregation analyses of family data suggest that the inheritance pattern closely approximates a single major locus with an autosomal dominant mode of transmission. These analyses on different sets of families have given very similar results suggesting genetic homogeneity at least in mode of inheritance. Under the most likely major locus model, not all individuals carrying a gene for TS actually express the disorder, or at least they do not display symptoms that lead to a clinical diagnosis of TS. The probability that individuals at risk for but not clinically affected by the disorder have actually inherited the genetic predisposition is influenced by their sex and age. By age 21 , most males have become affected if they are gene carriers for TS but only about $60 \%$ of female carriers have become affected. The pattern of symptoms also differs by sex. Obsessive compulsive disorder without TS, for instance, is more frequent in females than in males and appears to be an alternate expression of the disorder in females but not, or only rarely, in males.

About 500 loci have been tested for genetic linkage to a major locus for TS in the 14 multi-generational families our labs have been studying. Cell lines have been established on 312 individuals in the 4 New Haven-Rochester kindreds. Blood has been drawn on 385 individuals in the 10 families from the Netherlands and Norway collected by the Rotterdam group although it is only now in 1992 that funding has become available to establish cell lines from frozen lymphocytes. The Marshfield group has been routinely typing Dutch family 7 along with their own Wisconsin TS family and has also now typed one of the New Haven-Rochester kindreds for some microsatellite systems.

No strong, positive evidence of linkage has yet been found. In the case of a complex disorder like TS, a lod score of about +5 or higher would be the minimum positive evidence for linkage. As the number of independent marker loci tested rises, the minimum acceptable lod score should also be increased to compensate for the number of times a spurious positve lod score can occur by chance. One rule of thumb being followed to adjust for chance data configurations is to add the logarithm of the number of independent loci tested to the minimum positive lod score of 3 .

Assuming the same major locus underlies the disorder in the different kindreds we are studying, then our pooled results have excluded more than $80 \%$ of the autosomal genome with lod scores less than $\mathbf{- 2}$. These exclusions are based on a combination of pairwise and multipoint linkage analyses. All families have not been tested for all marker loci, however, and sometimes the same markers typed on different families have not been sufficiently informative to allow a useful test of linkage in all the kindreds.

When the linkage results are examined separately by family, the exclusionary totals are substantially less than $80 \%$ for each family and the particular chromosomal regions excluded vary across the families. If TS is genetically heterogeneous, then our search for linkage is not as far along as is implied by our pooled results.

However, those marker loci that have been typed on multiple families show no evidence for genetic heterogeneity at present. Thus, no subset of the kindreds under study has given strong, positive evidence of linkage when the remaining kindreds provide exclusionary findings in the same region.

Among the families collected for the New Haven and Rochester collaboration, large stretches of the genome have not been excluded yet on chromosomes $4,6,9,11,12,13$, $14,15,16,21$, and 22 . The Rotterdam study families have not yet excluded sizeable portions of chromosomes $1,2,3$, $4,5,6,8,10,14,17$, and 20 . The largest fraction of the genome excluded is about $45 \%$ in one of the New Haven study families and just over $50 \%$ in one of the Rotterdam study families.

Weakly positive lod scores (lods between 1 and 2) also have been observed near many markers in our families. We are targeting these areas in selecting additional markers to type.

This work has been supported in part by separate grants from the Tourette Syndrome Association to KKK, DLP, RK, JW, and BAO. Additional support has come from NIH grants: MH30929 and MH39239 to KKK; NS16648 and MHO0508 (ADAMHA RSDA) to DLP; and NS24278 to RK. 